\title{
Distortion Techniques for Sketching Interaction
}

\author{
Paul Schmieder* \\ Department of Computer Science, \\ University of Auckland, \\ Private Bag 92019, Auckland, New Zealand \\ psch068@aucklanduni.ac.nz, \\ \{beryl, john\}@cs. auckland.ac.nz
}

\begin{abstract}
Using sketching as the application domain, this research compares current distortion techniques for enlarging content. The goal is to develop a distortion lens which allows for a natural and uncomplicated drawing and writing experience on an electronic device.
\end{abstract}

Research Area: Distortion techniques to enlarge interaction spaces for ink.

\section{Introduction and Motivation}

The problem of limited display size on devices such as mobiles and laptops is well known and studied. Techniques that allow users to get a better overview or see more details such as pan and zoom (commonly referred to as overview and detail techniques) are very popular and widely applied [1], but are unsuitable for tasks where the complete content has to be visible at all times. By using distortion effects, focus and context techniques don't occlude content while showing zoomed parts in more detail [2].

The main problem when enlarging one area of a display is the additional space required for the enlarged part - perhaps occluding other parts. To avoid occlusion, focus and context techniques distort the content to generate the required space. For example, fisheye lenses $[3,4]$ use a central focus region for the detailed view with a distorted transition region surrounding it. The transition region generates the additional space required while providing a visual transition between the focus and context regions. Another distortion technique is sigma lenses [5] which use translucency to create a transition between the focus and context region. Sigma lenses do not require a separate transition region as the transition happens in the focus region thus saving valuable canvas space.

Distortion techniques have been the subject of studies for some time and have been proven useful, despite their disadvantages, in various domains such as viewing maps, selecting targets, and steering tasks [2, 7]. However, these distortion techniques have been evaluated for passive tasks, where content is viewed and selected, but not for active tasks of creation and editing. For the remainder of this paper the term 'active use' refers to creating and editing content and the term 'passive use' refers to viewing and selecting content.

* This dissertation work is supervised by Beryl Plimmer and John Hosking. 
The practicality and effectiveness of applying zooming techniques for active use for sketching has been shown by Agrawala and Shilman [8]. In their interface a region of the document is magnified to allow annotations to be written at the same size as the document's content. Their evaluation showed that zooming was useful when editing and writing in areas where space was limited, but participants found it less useful when sketching long gestures such as underlines and circles.

While Agrawala and Shilman [8] used a zoom region which covered parts of the content, Lank and Phan [9] employed a fisheye lens to avoid hiding context. Due to limited hardware performance Lank and Phan's lens remained at the position where it was activated rather than following the pen. Their use of a static lens helped them avoid the problems faced by movable lenses. Although their work is a first step toward the successful utilization of distortion techniques for active use, the static lens was found to be heavily constraining. It was identified in their evaluation study as the prototype's major drawback by a number of the participants [9]. Yet, the lens was, overall, perceived positively and despite this key limitation participants preferred the fisheye lens over having to scroll the canvas for their drawing tasks.

In summary, previous work has shown the usefulness of distortion techniques to tackle problems of limited space for passive use. Additionally, it indicates the potential for supporting interaction where content is created and edited although the realization is somewhat limited. These findings, in addition to my own experience in sketching[10, 11], led me to believe that new/novel distortion techniques can be of great benefit to better utilize limited canvas space for active use.

\section{Working Hypothesis}

Distortion techniques have potential for active use. In particular, the active use of distortion techniques is perceived positively by potential users and it results in performance gains. Additionally, the increased mental workload caused by distortion techniques does not hamper the user's creation and editing activities. To achieve this, current distortion techniques such as [3, 5-9] may have to be adjusted or a new technique developed such that their shortcomings for active use are resolved.

\section{Methodology and Conducted Research}

In this research we use digital sketching to investigate distortion techniques and their impact on the creation and editing of content. Sketching is an excellent application area as it provides users with an intuitive way to express and explore ideas. When designing a lens for more than just viewing and selecting content, there are many different aspects to consider. As distortion already puts additional mental workload onto the user we want to avoid using techniques that add to that workload. For example, we want to keep the distortion applied to a stroke being drawn to a minimum, especially the parts of the stroke close to the pen tip. For this research a sketch tool prototype has been implemented including different distortion lenses and several overview and detail techniques. 


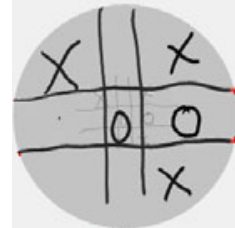

(a)

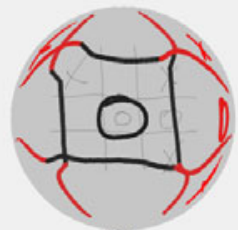

(b)

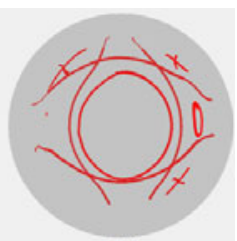

(c)

Fig. 1. Three different lenses showing a noughts and crosses game. Lens (a) is a magnifier. Lens (b) and (c) use a distorted transition region to generate the space required by the enlarged region. Lens (b) has a flat enlarged region while lens (c) has only a point at maximum magnification. Lenses (a) and (b) show the undistorted ink using translucency.

To determine which distortion techniques are suitable for active use and whether the current techniques have to be optimized or completely reworked, there are several steps to be taken. First, the task categories involved for active use have to be investigated to see whether each category requires a different lens; previous research indicates that different lenses are preferred by users when drawing and writing [8]. To study the different task categories, an evaluation study will be conducted where the participants are given drawing and writing tasks.

Second, distortion lenses and their configurations have to be developed for drawing and writing. Lenses can use different distortion techniques such as fisheye and sigma lenses. Within each technique there are a variety of options to choose from, such as the focus region being a single point or a flat area. The distortion function influencing the transition between the focus and context regions is another configuration decision. Once determined, many of these options can be fixed, though some options such as zoom level and size must be dynamically adjustable by the user. As there are complex relationships between the different options, a series of evaluation studies is necessary to answer these questions. Ultimately, the analysis of current distortion techniques may result in existing techniques being revised for active use or requiring a novel technique.

Third, having identified and implemented the preferred lenses, the lenses need to be compared to non-distortion techniques such as pan \& zoom and radar. The results will answer the main research question whether and which distortion techniques are suitable for active use. Therefore, a comparative study will be undertaken examining the performance of the different techniques in detail. Fourth, once the appropriateness of distortion techniques is confirmed, the lenses and their interplay have to be investigated and optimized. This includes the transitions between the lenses (e.g. when the user switches between drawing and writing) as well as how the lens is best operated (e.g. target acquisition) to optimize usage.

Since there is a considerable amount of work until we can answer our main research question we have conducted an informal study to see whether distortion techniques, even if not optimized, can compete with other techniques. We have asked students in our research group to complete a series of writing and drawing tasks using one lens as well as the radar and pan \& zoom techniques. Overall, they preferred using the fisheye technique but mentioned that the lens was less suitable for writing because it was too small. When parts of the word reach the transition region, these parts are distorted before one finishes writing the word, which users found confusing. These findings are similar to Lank's evaluation [9]. 


\section{Proposed Solution}

The lens will be designed according to the requirements described in the previous section. Specifically, the lens we propose will enlarge a part of the canvas while providing a transition between focus and context regions so that the complete content is visible at all times. Therefore, the lens may use newly developed distortion techniques or an optimized combination of already existing techniques. The lens will be optimized for active use and employ effective transitions between modes.

\section{Expected Research Contributions}

Our main research contribution will be to show the suitability or unsuitability of distortion techniques for active use. This includes known distortion techniques as well as novel techniques that may be developed in this research. We hope to show that the additional mental effort to comprehend the effect of distortion does not have a negative impact. Furthermore, we believe the methods used to transition between the distortion lenses, as well as the mode changes to control zoom and lens size will be applicable in other domains. Ultimately, the goal is that our lens toolkit will allow users to create and edit content faster and more conveniently than traditional overview and detail techniques on canvases where interaction space is limited.

\section{References}

1. Plaisant, C., Carr, D., Shneiderman, B.: Image-browser taxonomy and guidelines for designers. IEEE Software 12(2), 21-32 (2002)

2. Leung, Y., Apperley, M.: A Review and Taxonomy of Distortion-Oriented Presentation Techniques. ACM Transactions on Computer-Human Interaction (TOCHI) 1(2), 126-160 (1994)

3. Furnas, G.: Generalized fisheye views. ACM SIGCHI Bulletin 17(4), 16-23 (1986)

4. Spence, R., Apperley, M.: Data base navigation: an office environment for the professional. Behaviour \& Information Technology 1(1), 43-54 (1982)

5. Pietriga, E., Appert, C.: Sigma lenses: focus-context transitions combining space, time and translucence. In: Proceeding of the Twenty-Sixth Annual SIGCHI Conference on Human Factors in Computing Systems, pp. 1343-1352. ACM, Florence (2008)

6. Gutwin, C.: Improving focus targeting in interactive fisheye views. In: Proceedings of the SIGCHI Conference on Human Factors in Computing Systems: Changing our World, Changing Ourselves, pp. 267-274. ACM, Minneapolis (2002)

7. Carpendale, M., Montagnese, C.: A framework for unifying presentation space. ACM, New York (2001)

8. Agrawala, M., Shilman, M.: DIZI: A Digital Ink Zooming Interface for Document Annotation. In: Human-Computer Interaction, INTERACT 2005, pp. 69-79 (2005)

9. Lank, E., Phan, S.: Focus+Context sketching on a pocket PC. In: CHI 2004 Extended Abstracts on Human Factors in Computing Systems, pp. 1275-1278. ACM, Vienna (2004)

10. Schmieder, P.: Comparing Basic Shape Classifiers: A Platform for Evaluation Sketch Recognition Algorithms. In: Computer Science, p. 190. University of Auckland, Auckland (2009)

11. Schmieder, P., Plimmer, B., Vanderdonckt, J.: Generating Systems from Multiple Sketched Models. Journal of Visual Languages \& Computing (2009) 